\section{Resumen}

El presente artículo recoge los resultados y las reflexiones del análisis hecho a un material editorial diseñado por un centro interactivo de ciencia y tecnología, cuyo objetivo era divulgar el conocimiento científico y tecnológico sobre el gas natural.

Como estrategia metodológica, se realizó un análisis de contenido usando la herramienta propuesta por Fernández et al. (2002, 2003) sobre las visiones deformadas de la ciencia, lo cual se fortaleció con un abordaje en torno al proceso de diseño del material usando la Teoría Actor-Red.

Como resultado de la investigación, fue posible identificar que las deformaciones de la ciencia están íntimamente ligadas a las relaciones de poder que se configuran durante el proceso de diseño del material editorial. Paralelo a lo anterior, se identifica que el posicionamiento de los centros interactivos, como escenarios socialmente reconocidos en el campo científico y tecnológico, también tienen responsabilidades asociadas con las deformaciones de la ciencia y la tecnología en el contexto social en que actúan.

\section{Palabras clave}

Deformaciones de la ciencia, centros interactivos de ciencia y tecnología, material editorial para la enseñanza de la ciencia, teoría actorred.

\section{Abstract}

The article presents the research findings and the analytical reflections done with respect to a handbook designed and developed by an interactive center of science and technology in Colombia. The main objective of this material was to popularize scientific and technological knowledge about natural gas. We used the analytical tool proposed by Fernandez and collaborators $(2002 ; 2003)$ to identify the distorted images of science and technology presented in the handbook. These results were complemented and discussed using an actornetwork approach to analyze the design process of the handbook. This analytical approach allowed us to conclude that the distorted images of science and technology are intimately linked to the power relations which shape the design process of the educational material. Besides the latter, it was possible to identify that the social and political positioning of the interactive centers on science and technology also brings up social and political commitments which might contribute to enhance or destroy distorted images of science and technology localized in specific contexts.

\section{Key words}

Distorted images of science and technology, interactive centers of science and technology, educational material, actor network theory. 


\title{
¿De qué ciencia hablan nuestros materiales de divulgación?'
}

\author{
Manuel Franco Avellaneda² \\ Tania Pérez Bustos 3
}

\begin{abstract}
El mundo era tan reciente, que muchas cosas carecían de nombre, y para mencionarlas habia que señalarlas con el dedo. Todos los años, por el mes de marzo, una familia de gitanos desarrapados plantaba su carpa cerca de la aldea, y con

un gran alboroto de pitos y timbales daban a conocer los nuevos inventos.
\end{abstract} Fragmento de Cien años de soledad de Gabriel

García Márquez.

\section{Contexto de la investigación}

Investigaciones recientes sobre la enseñanza de las ciencias han determinado que una de las razones que explica la baja alfabetización, en ciencia y tecnología, es la imagen deformada de la tecnociencia que se transmite a las nuevas generaciones, principalmente a nivel escolar en los libros de texto y por medio de la relación pedagógica entre docentes y estudiantes (Abd-El-Khalick y Lederman, 2000). Al re-

\footnotetext{
${ }^{1}$ Texto recibido el 18 de marzo, evaluado el 11 de mayo y el 23 de junio y arbitrado el 30 de junio de 2009.

${ }^{2}$ Magíster en Educación, Universidad Pedagógica Nacional. Jefe de investigación y conceptualización en Maloka, programa de cobertura nacional con proyección internacional, de carácter cultural, educativo, científico, tecnológico, recreativo y turístico, que aporta a la construcción de una sociedad basada en el conocimiento, mediante el diseño de múltiples estrategias de apropiación social de ciencia y tecnología. mfrancoavellaneda@gmail.com

${ }^{3}$ Magíster en Estudios sobre el Desarrollo, Institute of Social Studies, Holanda. Estudiante del Doctorado Interinstitucional en Educación, Universidad Pedagógica Nacional, Universidad Distrital Francisco José de Caldas y Universidad del Valle como becaria de Colciencias. tpbustos@yahoo.com
} 
ferirse a visiones deformadas, esta literatura ha establecido que existen varias ideas o representaciones de la ciencia y la tecnología que se ponen en juego en los procesos de enseñanza y que éstas operan de manera simultánea, apoyándose unas a otras (Fernández et al., 2002; Gallego Torres, 2007).

En este sentido, retomando a Fernández et al. (2002), si bien el énfasis de esta investigación educativa ha estado en identificar la imagen empirista de la actividad científica y tecnológica en los textos escolares, es posible afirmar que esta visión usualmente está acompañada de concepciones que se apoyan, entre otras, en una subjetividad del científico, por lo general androcéntrica e individualista, que reproduce ideas de ciencia y tecnología neutrales y aisladas del contexto social, político, económico, y por tanto, capaces de dar cuenta de la realidad como tal, de manera universal, dogmática y rígida.

Un territorio poco explorado por estas investigaciones es la divulgación de la tecnociencia, particularmente la que tiene lugar en escenarios como museos interactivos o centros de ciencia y tecnología, más aún en aquellos que han emergido recientemente en países como Colombia, donde estos escenarios apenas están pensando cuál es su papel y articulación con el sistema educativo. Esto, a pesar de ser reconocidos como de gran aporte en la construcción de una percepción de la ciencia en el país (Aguirre, 2005; Daza y Arboleda, 2007). El objetivo principal de este artículo es ahondar en la reflexión sobre las visiones de la ciencia y la tecnología que son reproducidas por medio de materiales de divulgación producidos por estos escenarios.

El material impreso, y algunas veces virtual, que se construye en el centro interactivo sirve de apoyo para las visitas y tiene objetivos muy variados; La puede concebirse como herramienta de profundización, por lo que es usado para abordar los temas presentados en las exposiciones interactivas, ya que amplía la información sobre éstas, o, en algunos casos, propone experimentos, para realizar en casa o en la escuela, que están relacionados con la visita al centro interactivo. Otro objetivo común de estos materiales, es perfilarse como herramienta didáctica independiente, que puede ser utilizada en el aula apoyando y complementando el desarrollo de ciertas temáticas curriculares. También se pueden constituir en herramientas de información general sobre el centro interactivo, presentando mapas de recorridos, datos prácticos y generales sobre los módulos, entre otras.

El material editorial, analizado en esta investigación, corresponde a un programa llamado "Gas Natural en la Escuela" (en adelante GNE) que se llevó a cabo en el marco de un proyecto realizado por la empresa privada Gas Natural ${ }^{4}, \mathrm{Maloka}^{5}$ y la Secretaria de Educación Distrital de Bogotá (en adelante SED). El proyecto GNE se materializó en Maloka con tres estrategias: la construcción y puesta en escena de

\footnotetext{
${ }^{4}$ Gas Natural, tiene como mayor accionista al Grupo Gas Natural España, con una participación accionaria mayoritaria.

${ }^{5}$ Maloka es un centro interactivo ubicado en Bogotá (http://www.maloka.org).
} 
una estación interactiva compuesta por diversos módulos que abordaban el tema del Gas Natural, la presentación de una obra de teatro con títeres, en la que se abordaron aspectos sociales y de uso de este recurso, y una misión interactiva, realizada en las salas del centro interactivo, que estuvo apoyada por un material editorial ${ }^{6}$. Estas tres estrategias se complementaron con unos talleres realizados por el equipo de Maloka en las instituciones educativas.

El objetivo general del proyecto se orientó en el diseño e implementación de acciones que permitieran la enseñanza del gas natural como fuente de energía, haciendo referencia a sus aplicaciones, propiedades y beneficios, así como a los cuidados que se deberían tener en cuenta para su uso correcto. De manera particular, la cartilla objeto de análisis, se pensó como un material educativo sobre el gas natural para ser usado en el aula, que debía articularse a la misión interactiva que sucedía en la salas.

La cartilla está conformada por tres capítulos: Ciudad, Casa y Ecosistema. Para el análisis de las visiones deformadas que aquí presentamos, nos centramos en analizar el primero de estos capítulos. Cada uno de estos ejes temáticos estaba compuesto de cuatro partes: una de contexto, que hace un panorama de lo que se abordará en el capítulo; una segunda presenta una situación de reflexión, que para el caso del capítulo Ciudad, es la contaminación del aire; la tercera parte es un ejercicio de experimentación que sirve de propuesta a los niños para acercarlos a conocimientos científicos incluidos en la problemática de la segunda parte, para complementar, desde la experiencia, la reflexión allí propuesta; la última parte, es una reflexión sobre la temática abordada. Los contenidos están orientados por un personaje llamado Gasú, un dinosaurio que sirve como herramienta comunicativa en la cartilla.

Para el análisis del material editorial producido en el proyecto GNE, se utilizaron dos estrategias complementarias. Inicialmente, se analizó contenido de contenido del material, lo que permitió dar cuenta de las visiones deformadas de la ciencia y la tecnología allí presentes. Para ello, se siguieron las tesis planteadas, en ese sentido, por Fernández et al. (2002) y Gallego Torres (2007). En segundo lugar, y teniendo en cuenta que para estos autores los materiales educativos son asumidos como productos terminados y que, por tanto, no consideran sus procesos de construcción como dinámicas que contribuyen directamente en la configuración de dichas deformaciones, se incluyó como herramienta de análisis, de la presente investigación, la Teoría Actor-Red (en adelante TAR), que permite desentramar la manera en que esos objetos (módulos interactivos, material editorial, material didáctico) se construyen con fines educativos en el centro interactivo y se inscriben en relaciones de poder entre diferentes actores.

\footnotetext{
${ }^{6}$ La misión interactiva en las salas es una visita guiada que realizaron los escolares. Está descrita inicialmente como una misión, pues su objetivo es resolver un reto usando como herramientas los módulos interactivos.
} 
La premisa base es que tras el desarrollo de estos objetos existe una red de relaciones e intereses que pugnan y se materializan en mediaciones, temáticas, interacciones, etc. Su análisis permite visibilizar cuál es el papel político y educativo que cumplen los materiales en contextos determinados. Si bien el objetivo inicial de esta teoría busca dar cuenta de los desarrollos socio-tecno-científicos, en esta investigación se usa para analizar un escenario que se caracteriza por el diseño de materiales didácticos para la educación en ciencia y tecnología.

El presente artículo se divide en tres partes importantes: metodología, en la cual se explicita la manera en que se hizo la investigación; análisis de la información obtenida y, finalmente, conclusiones y recomendaciones para trabajos futuros.

\section{Metodología}

El estudio tiene, como punto de partida, la propuesta de Fernández et al. para analizar el contenido de textos educativos, a la luz de una serie de visiones deformadas de ciencia y tecnología promovidas por la enseñanza. Esta aproximación al material es complementada y problematizada con una reflexión desde la TAR. A continuación, presentamos cada una de estas aproximaciones metodológicas en detalle.

Se retomaron las siete visiones "deformadas" de ciencia de Fernández et al., y se identificaron una serie de criterios de análisis específicos que buscan facilitar la caracterización y el reconocimiento de cada visión en el material escogido. Estos criterios fueron extraídos de la lectura realizada por estos autores. Una revisión, de literatura relacionada con el tema, permitió complementar estos criterios y, en dos visiones en particular, llevó a la necesidad de incluir otros criterios no discutidos ampliamente por los autores aquí mencionados.

En total, se rastreó, en el material del proyecto GNE, la presencia de siete visiones y dieciocho criterios de análisis que permitieron reelaborar la matriz trabajada por Gallego (2007) y utilizarla como herramienta para identificar las visiones deformadas de la ciencia presentes en cómics.

El contenido del material GNE fue analizado identificando la presencia de las visiones en los tres niveles de categorización propuestos por esta autora: incidencia directa, incidencia indirecta (por omisión) y cuestionamiento de la visión. En el siguiente apartado, en el cual presentamos los resultados del análisis; la tabla 1 se refiere a las frecuencias con que estas visiones fueron encontradas a la luz de este análisis de contenido. A continuación, presentamos las categorías y los respectivos criterios de análisis definidos:

Visión socialmente descontextualizada. Esta visión hace referencia a la relación de la tecnociencia con su contexto social, político, económico y cultural, y a la manera en que esa relación define el rumbo del conocimiento científico. Esta categoría fue caracterizada por tres criterios de análisis. 1. Desconocimiento del contexto social externo de la ciencia, que implica una concepción de ciencia y tecnología cu- 
yos desarrollos no atienden a las necesidades y demandas sociales, ni se preocupan por los impactos sociales, culturales, económicos o ambientales que puedan causar. Asimismo, se enmarcaría, en este criterio, una concepción centrada en mostrar un contexto de emergencia favorable a la tecnociencia, con lo que se promovería una idea determinista de la misma.

2. Desconocimiento del contexto social interno de la ciencia, que implica pensar en una producción del conocimiento científico objetiva y completamente neutral, ajena a intereses y poderes internos de la propia comunidad científica. Este criterio fue tomado de la discusión realizada por Acevedo y Acevedo (2002) en relación con las creencias, en torno a la naturaleza de la ciencia, de profesores de educación secundaria. Y por último, 3. Consideración superficial de la tecnología, por cuanto se la ve como una mera aplicación de los conocimientos científicos, ignorando su papel en la construcción de dichos saberes.

Visión elitista. Esta visión promueve la idea de que el conocimiento tecnocientífico sólo puede ser producido por ciertos grupos sociales y en ciertos contextos. Esto significaría, en el caso colombiano, una ciencia y una tecnología, hecha, por un lado, por hombres rubios o de ojos rasgados pertenecientes al mundo desarrollado, y por otro, una idea de que sólo unos pocos cuentan con las condiciones cognitivas para acercarse a este conocimiento.

Esta visión puede desglosarse en cuatro visiones particulares: 1. Visión excluyente de la ciencia, por cuanto se concibe como de dominio de minorías especialmente dotadas, incluye discriminaciones de género, étnicas y socio-económicas. 2. Visión centrada en un estatus privilegiado del científico, desde la que se promueve una idea de una mayor jerarquía del conocimiento científico y del trabajo intelectual, frente a otros saberes, subvalorando el aporte de otros actores en el proceso de producción de conocimiento, como técnicos, maestros o usuarios. Este estatus privilegiado distanciaría a la ciencia y la tecnología de cualquier control democrático.

La relación que se establece aquí entre el estatus del conocimiento, la ciencia y tecnología, como parte de un proyecto democratizador, recoge las reflexiones posfeministas en torno a la ciencia y la tecnología, especialmente los planteamientos de Sandra Harding (1991) al rededor de la necesidad de pensar una ciencia más inclusiva, en términos sociales en la medida en que reconozca la necesidad de interlocución con otros saberes y otros sujetos de conocimiento.

3. Una visión inaccesible de la ciencia que circunscribe la construcción de conocimiento científico a ciertos escenarios que no tendrían un carácter público y abierto por naturaleza, como los laboratorios, y lo caracteriza como un conocimiento construido y difundido en un lenguaje especial que no es claro para quienes no pueden acceder a estos círculos cerrados. 4 . Una ciencia de corte individualista que reproduce una visión del conocimiento científico, como producto de grandes hombres que trabajan de manera aislada, ignorando el papel del trabajo colectivo, así como del intercambio entre equipos de trabajo. 
Visión empírico-inductivista. Destaca el papel de lo empírico y de la inducción en la construcción de un conocimiento científico tecnológico alejado de hipótesis y de elaboraciones teóricas. Se puede desglosar en tres elementos. 1. Énfasis en observación o experimentación, resalta el papel de la observación y la experimentación neutrales como base de la construcción de conocimiento. 2. Visión ateórica del conocimiento, que lo considera como producto resultado del azar y el descubrimiento espontáneo y casual. 3. Una ciencia que ignora la dimensión tecnológica de la producción de conocimiento, y que considera tales aportes como meramente instrumentales en el desarrollo de experimentos, favoreciendo un diseño mecánico de los mismos, y reforzando la concepción ateórica de la experimentación y del papel de la tecnología en la construcción del conocimiento (Fernández et al., 2003).

Visión algoritmica. Concibe la construcción de conocimiento como producto de un conjunto ordenado y finito de operaciones, que permite hallar la solución de un problema de manera precisa y objetiva; esta visión supone, de alguna manera, que el contexto de desarrollo de la ciencia y la tecnología se puede matematizar y predecir, desconociendo la incertidumbre, la ambigüedad o incluso la creatividad, como variables presentes en el contexto de desarrollo.

Está compuesta de dos elementos. 1. La legitimación de un método como producto de una serie definida de etapas a seguir mecánicamente (método científico), cuyo objetivo fundamental es dar cuenta de la realidad de manera exacta y objetiva (Acevedo y Acevedo, 2002) 2. Construcción rígida, destaca la rigurosidad y el proceso sistemático en la construcción del conocimiento, como principal camino hacia la objetividad, sin reconocer el papel activo que la transmisión y difusión de la ciencia y la tecnología cumplen en este proceso. Una visión rígida de la tecnociencia destaca el rigor del método y el tratamiento cuantitativo (ergo exacto) de los resultados obtenidos.

Visión dogmática. Esta visión, en estrecha relación con la anterior, promueve una construcción de conocimiento libre de problemas, tanto sociales como internos, de la propia tecnociencia y apoya una transmisión acabada de éste. Los criterios que definen esta visión de la ciencia y la tecnología son dos. 1. Concepción aproblemática del conocimiento que promueve una validación de éste por si mismo, sin relacionar su construcción y emergencia con necesidades sociales o problemas teóricos específicos; está apoyada en la transmisión de conocimientos ya elaborados, terminados y coherentes. 2. Concepción ahistórica de la construcción del conocimiento, que lo presenta de manera estática, sin considerar los procesos de cambio y transformación que han dado base a su construcción. Esta noción refuerza una imagen realista de la tecnociencia que no atiende a limitaciones, de allí su naturaleza dogmática.

Visión exclusivamente analítica. Esta visión da cuenta de una ciencia y una tecnología, centradas en la parcelación disciplinar y problemática para la construcción de conocimiento, lo que promueve su simplificación más que su naturaleza 
compleja, a la vez que desconoce los esfuerzos de unificación entre sus diferentes áreas. Se puede subdividir en dos componentes. 1. Ciencia no interdisciplinaria, que desconoce la participación de múltiples cuerpos de conocimiento en la comprensión de un problema y se centra en abordarlo desde disciplinas aisladas; 2 . Privilegio de la unicidad de la materia, lo que promueve una concepción unitaria de las teorías científicas y resalta su condición universal incuestionable. Esta concepción respalda la percepción de la tecnociencia como capaz de dar cuenta de una realidad.

Visión Acumulativa. Promueve una concepción de la construcción del conocimiento tecnocientífico, lineal y acumulativa, que evoluciona de manera fluida sin crisis ni cambios profundos. Los criterios que se han consolidado como característicos de esta visión son dos. 1. Énfasis en el crecimiento lineal, que implica una interpretación simplista y determinista de la evolución de los conocimientos científicos, asumiendo que se dan en forma lineal, sin rupturas; 2. Concepción históricamente aproblemática, desde la cual la construcción de conocimiento no puede dar lugar a dos paradigmas teóricos opuestos, por cuanto uno de ellos siempre logrará subsumir al otro. Esta visión es denominada por Acevedo y Acevedo (2002) como reduccionismo epistemológico.

Los criterios de análisis identificados para cada deformación, sirvieron como base para elaborar una matriz que permitió identificar ejemplos de las diferentes visiones en las secciones escogidas del material de divulgación; para ello, se consideraron aspectos de contenido (texto), ilustración y diagramación para cada caso. Los ejemplos se identificaron tomando unidades semánticas completas, para el caso del contenido, esto significa que no se escogieron frases de punto a punto, pues la divulgación trabaja bajo la premisa de que es mejor utilizar frases cortas, las cuales no siempre tienen una idea completa en torno a la tecnociencia.

En relación con la ilustración, se escogieron imágenes e historias completas, más que parceladas, y para la diagramación se identificaron contenidos o ilustraciones sobresalientes o subordinadas a la página en su conjunto. En la revisión de cada material se incluyó una columna adicional en la matriz, en la que se anotaron aspectos adicionales que no podían incluirse claramente en ninguna de las celdas dispuestas para la categorización. La tabla 1, en el apartado de análisis, resume los resultados del análisis de contenido efectuado utilizando esta matriz.

\section{Precisiones sobre la TAR y la manera, que fue usada para esta investigación}

El planteamiento central que recoge la TAR, parte de la existencia de un conjunto de relaciones de orden social, político y económico, que dan forma a los desarrollos tecnológicos, es decir, los modelan (Pinch, 1997, pp. 20-38). Esta base del planteamiento se desprende de lo que se ha llamado constructivismo social de la tecnología, una corriente de reflexión nacida en la década de los ochenta, dando origen a tres 
modelos ampliamente reconocidos: la construcción social de la tecnología ${ }^{7}$, la teoría Actor-Red y el modelo de sistemas 8 . Los planteamientos en común, de estos tres modelos, podrían resumirse en una metáfora que usa Hughes (Pinch, 1997, pp. 20-38) para describirlos como un "tejido sin costura", del que hacen parte, la tecnología, la sociedad, la política y la economía.

La TAR parte de un enfoque constructivista, en la medida que el Actor-Red se distingue del actor tradicional de la sociología por la inclusión de actores no-humanos (actantes) como elementos constitutivos de una red de mediaciones, que cuyo resultado es un producto técnico. Esto permite pensar en el papel que desempeñan estos actores (no-humanos) en nuestro estudio de caso, centrado en la cartilla que se diseñó para el proyecto GNE. Al respecto, la estrategia que propone la TAR consiste en "seguir" a estos actores en el proceso de conceptualización, diseño e implementación del material en un escenario dado, que, para el proyecto en cuestión, fueron algunos colegios de Bogotá, identificando cuál fue su participación en cada etapa y dando cuenta de los intereses que se pusieron en juego en cada momento.

Esta propuesta busca configurar una red compuesta por una serie de protagonistas heterogéneos (humanos y no-humanos) que se relacionan por periodos de tiempo y tienen intereses distintos en relación con el proyecto. Desde esta perspectiva, los artefactos forman parte del escenario social no sólo como materia inerte, sino como actores que lo transforman, pues encarnan una cadena de participantes que los modelaron, tanto en lo material (madera, metal, pintura, etc.), como en su función (colores llamativos, ajustarse a la mano derecha y no a la izquierda, para adultos o para niños, etc).

Cada vez que nos enfrentamos al uso un objeto no estamos en el principio, sino al final de una larga cadena de mediaciones que ocurren entre humanos y nohumanos, las cuales transforman nuestra visión convencional de un escenario social centrado en sujetos (Latour, 1998, pp. 249-267), pero, ¿cuáles son las mediaciones que suceden entre unos y otros actantes?, ¿cómo se configuran? Y ¿cómo configuran los espacios sociales en los que se desarrollan dentro del estudio de caso que nos interesa?

Para el caso particular del material investigado, la TAR se utilizó para problematizar los resultados del análisis de contenido, en el que se rastrearon las visiones defor-

\footnotetext{
${ }^{7}$ Sus principales conceptualizadores son Trevor Pinch y Wiebe Bijker, y sus planteamientos se pueden recoger a nivel general de la siguiente manera, según Ayus (2002): “a) se centra en la construcción social de los significados tecnológicos; b) en las trayectorias (o biografías) sociales de los artefactos tecnológicos y c) en los procesos interpretativos de re-significación y sus tensiones inherentes para hacer prevalecer o cohabitar un punto de vista sobre los artefactos técnicos; d) la dimensión de la representación social y las percepciones sociales son cruciales para el análisis social de la tecnología”.

${ }^{8}$ Este modelo fue propuesto por Thomas Hughes en la década del ochenta para el estudio de los sistemas socio-técnicos. En general según Osorio (2005): "permite mostrar con mayor detalle la interacción entre las partes y el todo, propio de una noción sistémica y, por otro lado, nos da una cierta idea de la dinámica de la tecnología”.
} 
madas en torno a la ciencia. Esta aproximación permitió dar cuenta de la manera en que estas visiones son producto de las relaciones de poder que se tejen entre actores humanos y no-humanos que participantes en el proceso de diseño de la cartilla.

El seguimiento nos permitió identificar los procesos de articulación y delegación de funciones educativas entre unos y otros actores, así como dar cuenta de las simplificaciones construidas por aquellos que, además de ser "diseñadores", se convierten en "pedagogos" durante el diseño del material. Por otra parte, el ejercicio brindó herramientas para visualizar el papel que el proyecto GNE, representado en la cartilla, cumplió para cada uno de los participantes en este proceso, lo que permitió matizar y contextualizar los resultados de análisis de contenido en el que se rastrearon las visiones deformadas.

Para la configuración de la red, de manera que sirviera de base para el análisis de la TAR, se hizo seguimiento a la manera en que se han configurado las relaciones, sociales y de poder, que se propician desde el centro interactivo por medio del material editorial señalado. Se buscó identificar, particularmente, qué actores humanos y no-humanos estructuran la red en torno al proyecto GNE y la cartilla que se diseñó en ese marco, así como las mediaciones que se establecen entre ellos y la manera en que concibe el conocimiento científico tecnológico en relación con el gas. Asimismo, se abordó un análisis acerca de la naturaleza de los principales actores, buscando identificar el contexto macro en el que se inscribe el proyecto y su accionar educativo.

Metodológicamente, se realizaron entrevistas a los distintos participantes, de manera que nos permitieran describir la red de actores desde cada perspectiva particular. En este ejercicio fueron entrevistados, en diferentes momentos del proceso de diseño, tres maestros, que participaron en el diseño de la cartilla, y quince estudiantes de las instituciones educativas participantes, así como dos profesionales involucrados en el diseño de Maloka, dos funcionarios de Gas Natural, la persona encargada del proyecto desde la Secretaria Distrital de Educación, y un experto en gas natural.

Se tuvieron en cuenta tres aspectos importantes: los momentos y actores que ellos identificaban en el proceso de diseño, así como las diferentes perspectivas que

\footnotetext{
${ }^{9}$ Michel Callon (1998) propone que los ingenieros y científicos se convierten en sociólogos cuando trabajan en desarrollos de alta innovación, en la medida en que, además de resolver problemas del mundo natural, tienen que construir interpretaciones del funcionamiento, necesidades y configuraciones del mundo social, esto permite que, al abordar las interpretaciones propuestas por ellos, se pueda ver una buena parcela del escenario social que están ayudando a configurar. Para este caso específico hacemos el símil con los pedagogos, pues los diseñadores (científicos, ingenieros, comunicadores etc.) construyen visiones educativas que podrían usarse para evaluar el escenario que ayudan a construir, de hecho, proyectos como Pequeños Científicos, Maloka, El Museo de la Ciencia y el Juego, Buinaima y Explora, entre otros, que se han concebido principalmente por científicos de ciencias naturales y técnicas que han construido visiones del entorno educativo al cual quieren afectar, con una baja o muy poca participación de pedagogos formados.
} 
cada uno tuvo sobre la meta propuesta (su objetivo para participar en el proyecto y en el diseño de la cartilla). En segundo lugar, el papel que desempeñó cada actor en el proyecto y, finalmente, saber que cada actor aportó en el proceso de diseño de la cartilla, lo cual fue socializado con la reflexión acerca de visiones deformadas que se mencionó anteriormente y permitió contextualizar los resultados producto de este análisis.

El ejercicio de visualizar momentos y actores permitió identificar qué visión del proyecto y qué momentos del proceso de diseño de la cartilla reconocía cada actor. De esta manera, fue posible construir un mapa de relaciones que nos dio las bases para esquematizar la forma en que el proyecto fue concebido por éstos y qué lugar tuvo en él la cartilla; la gráfica está representada en la figura 1. Las definiciones se pudieron contrastar y problematizar con las definidas previamente por Maloka. Igualmente, hicieron visibles las diferentes estrategias que se siguieron, desde cada caso, en la consecución del objetivo propuesto con la cartilla.

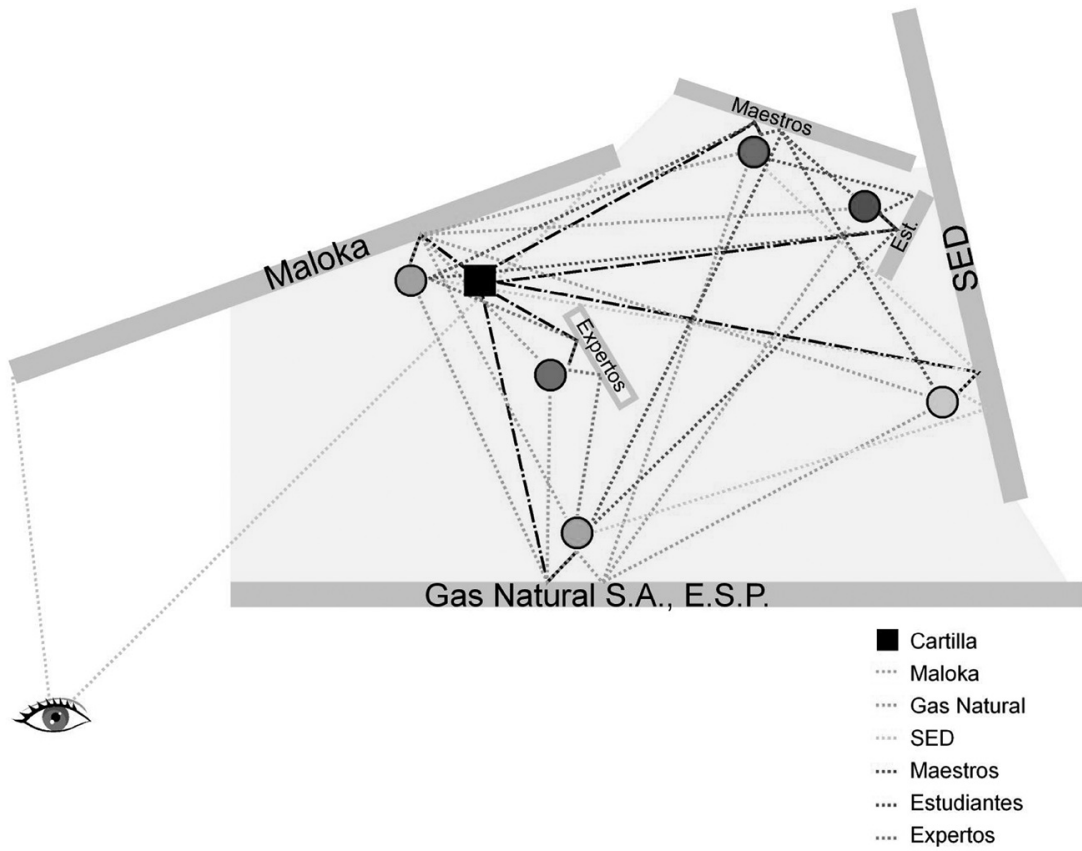

Para la realización de la imagen, se tuvieron en cuenta los actores participantes en el proyecto, representados con barras grises (a manera de espejos) y los puntos (frente a cada barra) simbolizan las personas entrevistadas (pertenecientes a cada grupo de actores). En el proceso de investigación, cada actor hizo visibles a otros protagonistas, mientras que a otros no los identificó como partícipes del proyecto. 
Lo anterior permitió construir la figura presentada, que da cuenta de este mapa de relaciones. Por ejemplo, si se siguen las líneas punteadas tienen su origen en cada uno de los puntos, se puede notar que mientras Maloka es vista por todos los actores, los estudiantes y expertos son invisibles entre ellos y para la Secretaría de Educación. Por otra parte, solo una persona externa al proyecto identifica a Maloka como actor central del mismo (lo cual está señalizado en la figura con un ojo). Nótese la manera en que la cartilla (identificada con un cuadrado negro) es referente para todos los actores entrevistados, hecho que permite mostrar su protagonismo en el proceso.

En la medida en que se establecieron los momentos del diseño, también fue posible encontrar otros actores que configuran la red. Con ayuda de las entrevistas realizadas, inicialmente a actores de Maloka, Gas Natural y SED, quienes en principio se reconocieron como protagonistas del proyecto, se lograron identificar otros actores que ellos visualizaron como participantes, así como el papel que éstos tuvieron desde su perspectiva (docentes, estudiantes, expertos).

En segundo lugar, se identificó la documentación existente sobre el proyecto, lo que permitió dar cuenta de su historia. Este ejercicio hizo posible recoger los antecedentes del proyecto y de los actores; el análisis de estos documentos se centró en dar cuenta de las intenciones de los actores (de cómo éstas enmarcan el para qué y el cómo de la propuesta educativa) y de manera particular cómo se inscribe en ellos el conocimiento científico tecnológico, en este caso sobre el gas natural y la energía fósil (¿qué temas son priorizados?, ¿cómo y por qué aparecen priorizados?, ¿cómo estas temáticas van transformándose, o no, durante el proceso?, ¿quiénes orientan tal transformación y hacia dónde?). En este punto fue de vital importancia rastrear la existencia de planteamientos críticos frente a estos contenidos y si operan con éxito o si son cooptados por intencionalidades comerciales y la manera en que esto ocurre.

\section{Análisis del material}

A continuación, presentamos los resultados obtenidos del análisis de contenido hecho al material, a la luz de la matriz de identificación de visiones deformadas de ciencia y tecnología antes explicada; ponemos en diálogo estos resultados con la visión del proceso de diseño que nos da el trabajo de seguimiento realizado a los actores a la luz de la TAR.

\section{Visiones sobre la ciencia y la tecnología presentes en el material de divulgación}

La cartilla, en general, combate las visiones deformadas, pues el 63,1\% de los ejemplos identificados en su contenido se ubica en el campo de la no incidencia, mientras que el $11,2 \%$ incide de manera directa y el $25,7 \%$ no hace ningún tratamiento de las mismas, lo cual podría interpretarse como una incidencia indirecta. 
Viendo el material como producto terminado, desde las categorías de Fernández et al., éste combate deformaciones, en la medida en que se contextualiza socialmente la producción del conocimiento, no se promueve una visión empírico-inductivista de éste y se esfuerza por problematizar su visión algorítmica y elitista. Al tiempo, es posible como, a pesar de promover visiones alternativas a las mencionadas, la cartilla incide de directamente en una visión dogmática del conocimiento científico. Las tablas 1 y 2 resumen los resultados de las visiones encontradas en este sentido, cabe aclarar que estas tablas dan cuenta de las frecuencias con que las diferentes visiones fueron identificadas en el contenido de la cartilla y que este ejercicio de rastreo fue hecho por los investigadores de manera previa al uso de la TAR.

TABLA 1. PORCENTAJE DE INCIDENCIA POR LAS VISIONES CONSOLIDADAS (GNE)

\begin{tabular}{|l|c|c|c|}
\hline \multicolumn{1}{|c|}{ Visión } & $\begin{array}{c}\text { Incidencia } \\
\text { Directa. }\end{array}$ & $\begin{array}{c}\text { Incidencia } \\
\text { Indirecta. }\end{array}$ & Combate \\
\hline Descontextualizada & $1,0 \%$ & $4,9 \%$ & $27,2 \%$ \\
\hline Elitista & $0, .0 \%$ & $1,5 \%$ & $8,7 \%$ \\
\hline Empírico-inductivista & $2,9 \%$ & $1,9 \%$ & $6,8 \%$ \\
\hline Algorítmica & $2,9 \%$ & $1,5 \%$ & $8,7 \%$ \\
\hline Dogmática & $4,4 \%$ & $11,7 \%$ & $5,8 \%$ \\
\hline Exclusivamente analítica & $0,0 \%$ & $2,9 \%$ & $5,8 \%$ \\
\hline Acumulativa & $0,0 \%$ & $1,5 \%$ & $0,0 \%$ \\
\hline Total & $11,2 \%$ & $25,7 \%$ & $63,1 \%$ \\
\hline
\end{tabular}

\begin{tabular}{|c|l|c|c|c|}
\hline Visión & \multicolumn{1}{|c|}{ Característica } & $\begin{array}{c}\text { Incidencia } \\
\text { Directa. }\end{array}$ & $\begin{array}{c}\text { Incidencia } \\
\text { Indirecta. }\end{array}$ & Combate \\
\hline \multirow{2}{*}{$\begin{array}{l}\text { Descontex- } \\
\text { tualizada }\end{array}$} & Socialmente neutra (CE). & $1,1 \%$ & $2,2 \%$ & $24,2 \%$ \\
\hline & $\begin{array}{l}\text { Socialmente neutra (CI). } \\
\text { Consideración superficial }\end{array}$ & $0,0 \%$ & $2,2 \%$ & $4,4 \%$ \\
\hline \multirow{2}{*}{ Elitista } & de tecnología. & $0,0 \%$ & $1,1 \%$ & $2,2 \%$ \\
\hline & Excluyente. & $0,0 \%$ & $1,1 \%$ & $5,5 \%$ \\
\hline & Estatus privilegiado. & $0,0 \%$ & $1,1 \%$ & $1,1 \%$ \\
\hline & Inaccesible. & $0,0 \%$ & $0,0 \%$ & $1,1 \%$ \\
\hline
\end{tabular}




\begin{tabular}{|c|c|c|c|c|}
\hline \multirow{3}{*}{$\begin{array}{l}\text { Empírico - } \\
\text { Inductivista }\end{array}$} & $\begin{array}{l}\text { Resalta la empiria como } \\
\text { base de método. }\end{array}$ & $2,2 \%$ & $0,0 \%$ & $0,0 \%$ \\
\hline & Ateórica. & $0,0 \%$ & $1,1 \%$ & $4,4 \%$ \\
\hline & $\begin{array}{l}\text { Ignora la dimensión } \\
\text { tecnológica. }\end{array}$ & $1,1 \%$ & $1,1 \%$ & $3,3 \%$ \\
\hline \multirow{2}{*}{ Algorítmica } & Legitima un método. & $1,1 \%$ & $0,0 \%$ & $0,0 \%$ \\
\hline & Rígida. & $1,1 \%$ & $1,1 \%$ & $6,6 \%$ \\
\hline \multirow{2}{*}{ Dogmática } & Aproblemática. & $3,3 \%$ & $6,6 \%$ & $3,3 \%$ \\
\hline & Ahistórica. & $0,0 \%$ & $2,2 \%$ & $1,1 \%$ \\
\hline \multirow{2}{*}{$\begin{array}{l}\text { Exclusivamente } \\
\text { analítica }\end{array}$} & No interdisciplinaria. & $0,0 \%$ & $0,0 \%$ & $4,4 \%$ \\
\hline & $\begin{array}{l}\text { La unicidad versus } \\
\text { la diversidad. }\end{array}$ & $0,0 \%$ & $2,2 \%$ & $0,0 \%$ \\
\hline \multirow{2}{*}{ Acumulativa } & Crecimiento lineal. & $0,0 \%$ & $0,0 \%$ & $0,0 \%$ \\
\hline & Aproblemática. & $0,0 \%$ & $1,1 \%$ & $0,0 \%$ \\
\hline Total & & $10 \%$ & $23 \%$ & $67 \%$ \\
\hline
\end{tabular}

\section{Problematización de las visiones encontradas en la cartilla a la luz de la TAR}

Profundizaremos sobre los resultados, buscando contextualizarlos a la luz del seguimiento hecho a los actores, usando la TAR. Con este ejercicio, se busca problematizar lo encontrado en el análisis basado en la propuesta de Fernández et al., ya que nos permitirá señalar que el contexto de relaciones de poder, que enmarcan el diseño de la cartilla, además de dar la impresión de promover una visión no-deformada del conocimiento en torno al gas natural como recurso energético, también promueven una visión neutral frente a éste, lo que termina posicionándolo como mejor alternativa frente a otros combustibles y lleva a que el objetivo educativo de la cartilla para Maloka y la SED se traduzca en un objetivo de mercadeo para Gas Natural.

Según lo planteado, el material está combatiendo principalmente la visión de un conocimiento científico socialmente descontextualizado $(27,2 \%)$, que se sustenta en su propuesta gráfica y de contenidos, según la cual el problema de la energía aparece situado en un contexto particular: Bogotá y sus problemas de contaminación (figura 2). Esta apuesta por contextualizar va a ser reconocida por los maestros que participaron del diseño de la cartilla como de gran valor pedagógico, pues permite que los estudiantes piensen en un problema de orden ambiental desde su realidad cotidiana. 


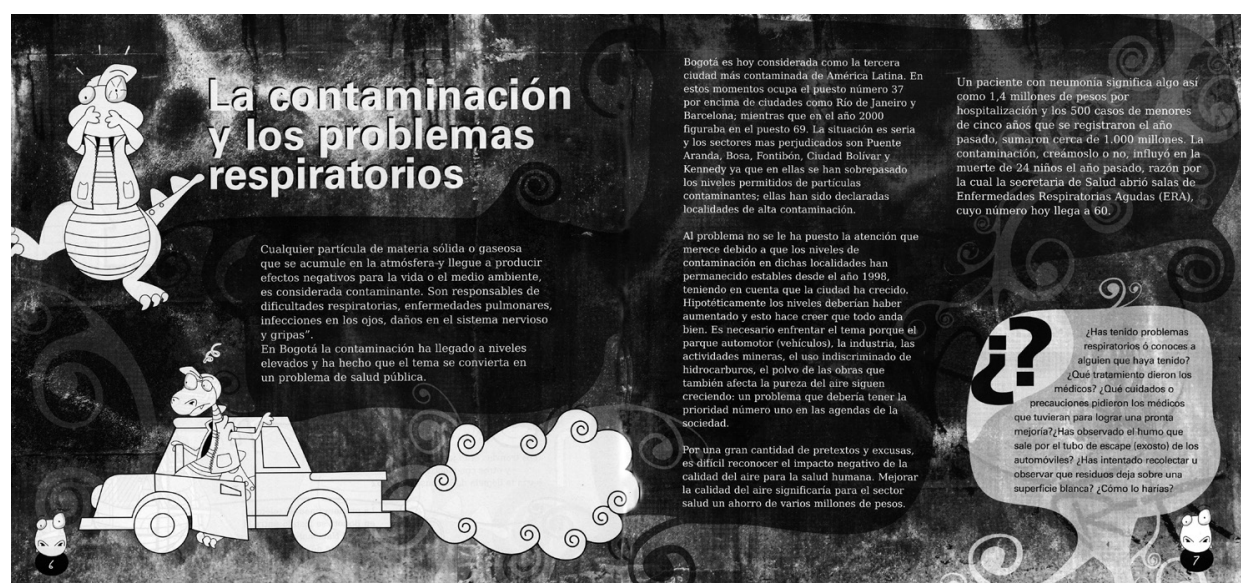

Al analizar detalladamente la cartilla, encontramos que esta contextualización pedagógica respalda la idea según la cual el gas natural es la mejor alternativa frente al problema de contaminación urbana. Es decir, la contextualización se encuentra al servicio de la promoción de un recurso energético frente a otros. Esta complejización problemática de la contextualización, que no parece ser visible para los maestros, se resalta cuando se hace seguimiento al actor Gas Natural, partícipe del diseño de la cartilla, y a sus intereses. Desde allí, encontramos que esta empresa busca posicionar el Gas Natural como energía "limpia” por medio de este material educativo.

Es útil, en este sentido, retomar las palabras del funcionario de Gas Natural, E.S.P refiriéndose a las ventajas de trabajar con Maloka: “[...]Maloka no sólo se considera un escenario de ciencia y tecnología, sino también tiene amplio reconocimiento en el campo medio ambiental, desde esa perspectiva nuestro producto encaja muy bien, pues nuestro producto es limpio y amigable con el medio ambiente" (Funcionario GN 1, enero de 2008). Este referente permite problematizar la manera en que se expone la realidad de la contaminación, en la cartilla que hace énfasis en los problemas del diesel y en especial en el material particulado que éste genera en la atmósfera.

Sin embargo, esta descripción de la contaminación, en apariencia neutral, deja de lado el problema, causado por la mala combustión de los autos que usan gas natural, de la liberación de metano en la atmósfera ${ }^{10}$. Lo que nos interesa resaltar aquí es la manera en que esta omisión en la cartilla contribuye al posicionamiento

\footnotetext{
${ }^{10} \mathrm{Si}$ bien los residuos, producto de la combustión del Gas Natural, son menores en comparación con los causados por el uso de gasolina o diesel, no por ello deja de liberar residuos a la atmósfera, lo cual en cualquier caso irá en aumento en la medida en que el parque automotor que use gas aumente.
} 
social del gas natural (en contraposición a otros recursos energéticos) y que esto es producto de una contextualización social que de algún modo se realiza de manera acrítica.

Esta búsqueda de posicionamiento del producto, y la cartilla como estrategia para ello, también puede explicarse a la luz de otro de los resultados encontrados con el análisis inicial, que señala cómo la cartilla parece promover una idea no elitista y creativa de la producción de conocimiento $(8,7 \%)$, aspecto que también será considerado de gran valor pedagógico, tanto por los maestros como por los expertos partícipes en el diseño del material. Como respaldo a esta apuesta por incluir al lego como actor de la construcción científica, la cartilla presenta imágenes de estudiantes (caracterizados en el personaje) haciendo experimentos con la ayuda de adultos (figura 3) ${ }^{11}$, así como una diversidad de papeles sociales que contribuyen de manera positiva o negativa a los problemas ambientales (figura 4).

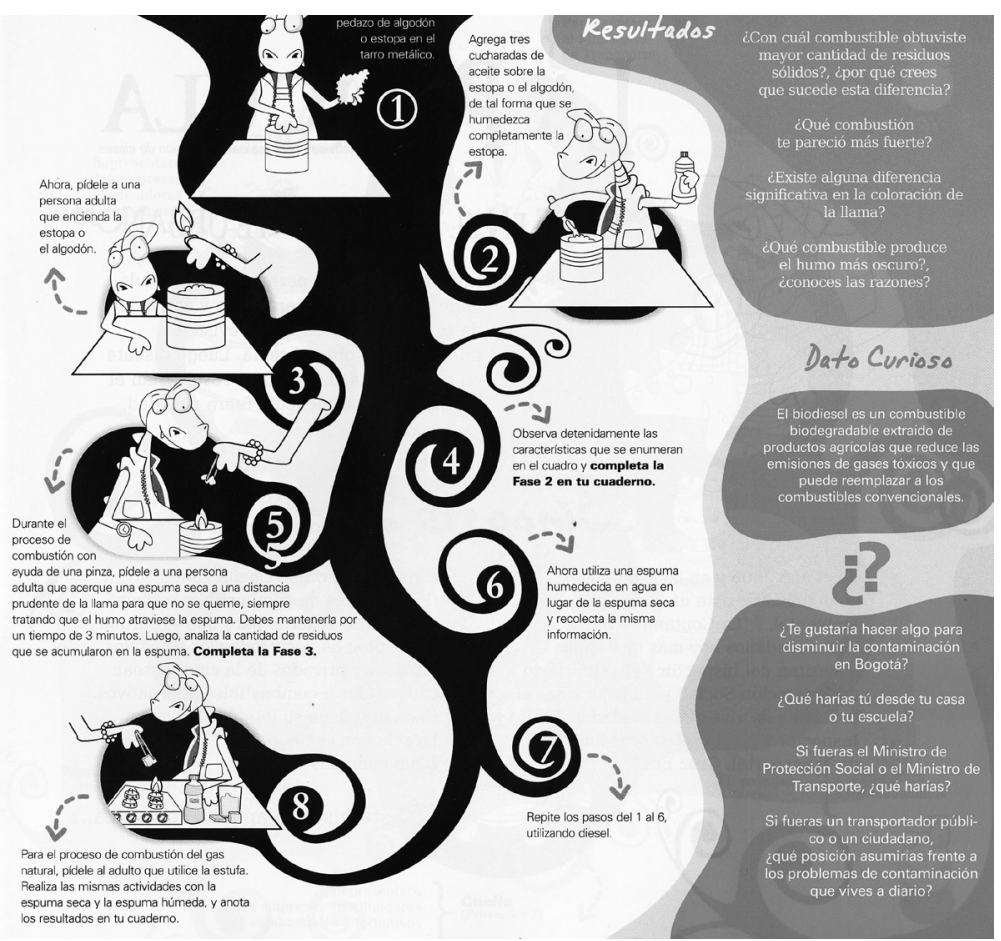

\footnotetext{
${ }^{11}$ La cartilla no muestra a un adulto, en el caso del capítulo de ciudad, aparece el brazo de una mujer que realiza los pasos que tienen mayor peligro, como encender la llama o exponer la espuma al fuego.
} 


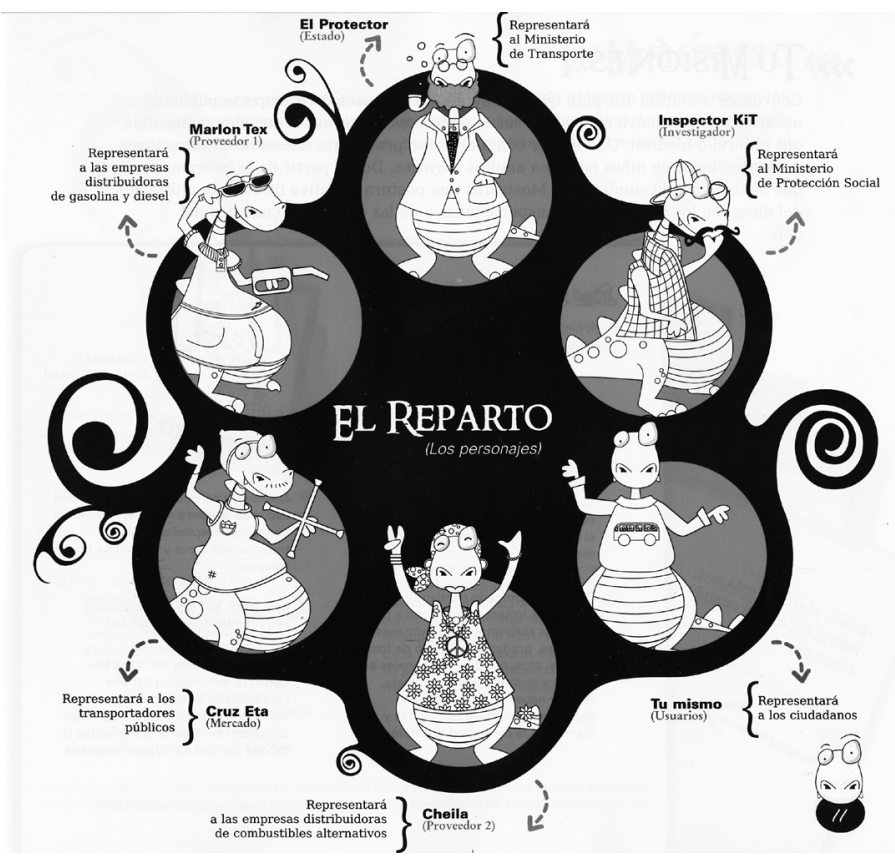

Sin embargo, la visión del lego como partícipe también tiene una carga de neutralidad promovida por Maloka, que es aprovechada nuevamente desde los criterios publicitarios de la empresa. Así, encontraremos que Gas Natural, buscará que se promueva una imagen del público como usuario calificado, que asegura que no ocurran accidentes en el hogar, lo que potencialmente deterioraría la imagen del gas natural como recurso. En este sentido, una de las integrantes del equipo de Maloka dice:

[...] Con relación a Gas Natural y a las negociaciones, pues ellos nos decían qué cosas no podían ir, por ejemplo: en los muerticos por el gas, nosotros negociamos, pues se tenía que decir el problema, también era necesario que ellos comprendieran que había parte de los intereses de Gas Natural pues la gente necesita saber qué puede pasar y que evite que pase, entonces ellos aceptan eso (Funcionaria 2 MK, marzo de 2008).

Diremos aquí que lo aceptan, pues va en beneficio de su imagen; en otras palabras, constituye un reconocimiento pragmático instrumental del saber lego, no en un reconocimiento que tenga como punto de partida el diálogo con sus conocimientos cotidianos.

La idea de la cartilla como medio para el posicionamiento del gas natural, que se apoya en el reconocimiento instrumental del estudiante como usuario, también 
puede respaldarse al problematizar lo encontrado en el análisis inicial desde los procesos de la TAR, pues pareciera combatir una idea de conocimiento algorítmico $(8,7 \%)$. Esto se hace evidente, en la cartilla, con una invitación al lector a investigar y proponer soluciones frente a problemáticas relacionadas con el mal uso y aprovechamiento de temas energéticos en su contexto inmediato, lo que es comprendido por los maestros y por el equipo de Maloka como un ejercicio de involucrar a los estudiantes en la resolución de problemas. Sin embargo, la manera en que se presenta el ejercicio, orienta la investigación autónoma, en tanto que la dirige a indagar por cómo el gas es una de las mejores alternativas para mejorar estas condiciones problemáticas de la cotidianidad, de modo que tras la investigación pareciera esconderse una orientación de marketing de la empresa.

Otro de los aspectos encontrados en el análisis inicial, y que resalta la relación con las visiones de ciencia que el material de GNE combate, es su tendencia a presentar una idea de ciencia alejada de lo empírico-inductivista $(6.8 \%)$, lo cual se haría visible porque el material se esfuerza por realizar un reconocimiento explícito de la dimensión tecnológica, en relación con el gas natural como recurso energético. Esto es evidente en la manera en que se propone a los estudiantes realizar el experimento sobre la combustión del gas, allí se plantea la observación detallada de tres combustibles diferentes con el objeto de caracterizarlos, para luego formular hipótesis en torno a dicha caracterización.

Si bien esta visión no es significativamente importante respecto de las anteriores, podría explicarse a la luz de dos circunstancias en la construcción de la red de actores por un lado, Gas Natural se considera una empresa tecnológica, poseedora de un conocimiento que le permite ser líder en el campo, y busca, como señalábamos anteriormente, ser reconocida por ello y transmitir este mensaje por medio de la cartilla; por otro lado, se encuentra como el personal de Maloka, encargado de liderar el desarrollo de la cartilla y la conceptualización de las actividades, estuvo conformado por ingenieros (el principal actor experto también tiene esta formación).

Lo que llama la atención de este resultado es que el énfasis de lo tecnológico y la construcción del conocimiento, a partir de las reflexiones de los estudiantes, al tiempo que propone otros modos no metódicos de alcanzar el conocimiento, se convierte en una manera de neutralizar el papel político de Gas Natural, situación que de algún modo refuerza la manera en que la empresa privada se convierte en aliada incondicional del centro interactivo, no sólo porque permite reconocer sus propios desarrollos como pertenecientes al campo de la producción de conocimiento, sino porque al hacerlo, como hemos señalado, neutraliza sus intereses económicos, sociales y políticos.

En relación con la incidencia indirecta del material en la promoción de ciertas visiones, el análisis inicial muestra que esto es particularmente cierto para el caso de la visión dogmática del conocimiento $(11,7 \%)$, la cual está caracterizada por una mirada aproblemática de ciertas preguntas en torno a las actividades propuestas $\mathrm{y}$, 
en algunos casos, por la descontextualización histórica de algunos conceptos y realidades respecto al gas natural.

En este sentido, es importante mencionar que todo el texto está basado en mantener unos hábitos y necesidades relacionados con el transporte, que no van a ser problematizados, centrándose en la pregunta por los combustibles. La problemática de la contaminación no toca a los usuarios, cuya única tarea pareciera la elección entre un combustible u otro. Esta visión dogmática, aproblemática de la contaminación, refuerza la idea del lector de la cartilla como consumidor de combustible y no como participante activo de una realidad socio-técnica.

De manera complementaria a esta visión, del estudiante-público como usuario-consumidor, el análisis inicial alertó sobre la ausencia del científico, el cual incluso no está en la controversia planteada sobre el transporte, sin contar con la ausencia de los maestros, quienes tampoco aparecen en ninguna parte del texto. Finalmente, aparece otro elemento interesante, la imagen femenina que representa a las empresas de combustibles “alternativos", en este caso gas natural y biodiesel. En lo que sigue, profundizaremos sobre estas representaciones e invisibilizaciones, a la luz del proceso de seguimiento que se hizo a la red de actores que participaron del material.

La ausencia del científico en la controversia que plantea la cartilla parece suponer una visión de neutralidad de este actor y, de alguna manera, de su conocimiento. Esta neutralidad podría tener relación directa con la delegación, de todos los actores, al centro interactivo como conceptualizador y poseedor de un conocimiento científico y tecnológico; en este sentido, no colocar la posición del científico o del ingeniero (o neutralizarla a partir de la experimentación abierta del niño) permitiría proteger la imagen de este actor o instrumentalizarla en beneficio de la imagen de la empresa, que, de alguna manera, el centro interactivo está representando. Esto va a ser igualmente evidenciado en la aparición de el centro interactivo como principal protagonista del material, mientras que los expertos y su papel en la conceptualización del mismo no serán visibles en los créditos de la cartilla.

En relación con la ausencia de los maestros en el material, es necesario decir que ésta es especialmente paradójica, dado que los maestros son especialmente reconocidos como unos de los principales actores del proyecto y del proceso de diseño por parte de Maloka, Gas Natural y la SED. Es aún más extraño si consideramos que la cartilla estaba pensada para ser usada en el aula. Al respecto, los integrantes del equipo del centro interactivo llegan incluso a considerar que algunas de las actividades propuestas sólo se pueden realizar en el salón de clases. De hecho, en la cartilla la actividad llamada "La película: caos en el transporte urbano" tiene una aclaración en relación con esta circunstancia.

Esta aparente contradicción que se desconoce el papel del maestro, a pesar de asumir que las actividades se realizarán en el escenario escolar, puede explicarse a partir de tres elementos observados en el proceso: el primero, el interés sobre el 
aprendizaje que tiene Gas Natural y el centro interactivo, es decir, sobre lo que los niños harían, experimentarían y lograrían, lo que explicaría el protagonismo de éstos, que se evidencia claramente en toda la cartilla. El segundo, relacionado con la falta de instrucciones para los maestros; al respecto dice una de las integrantes del equipo de Maloka:

\section{[...] El piloto también se hizo para mirar si las instrucciones que dábamos en la cartilla eran suficientes para los profesores que iban aplicar las actividades, [...] pero frente a la situación que se dio en el que los profes nos dejaban solos en el piloto, mostraba que no eran suficientes las instrucciones y que faltaba mucho para que ellos entendieran cuales eran los objetivos (Funcionaria 2 MK, marzo de 2008).}

Situación que fue obviada por el centro interactivo, con lo que la cartilla terminó imprimiéndose sin modificaciones, al punto que en ninguna parte se dan orientaciones para el maestro; tan sólo se llama la atención en ciertas actividades sobre la necesidad de contar con la ayuda de un adulto. por último, los propios maestros reconocen explícitamente al centro interactivo como poseedor de un conocimiento del que ellos son subsidiarios, incluso un conocimiento de tipo didáctico y pedagógico, con lo que ocultan su papel y, de alguna manera, aceptan las decisiones que toma el centro interactivo respecto del material, así como de lo que ocurre en el aula, circunstancia que expresa una de las maestras participantes, “[...] alguien me dijo: ¡Oiga pero usted no aparece acá! (refiriéndose a la cartilla), yo le dije: pero igual yo no hice nada ahí" (Docente CRH, marzo de 2008).

Finalmente, la imagen femenina que representan las empresas de combustibles alternativos (figura 4), puede estar relacionada con la imagen que Gas Natural quiere posicionar como empresa amiga del medio ambiente, ya mencionada en este apartado. En este sentido, pensar en un personaje de caracterización hippie e incluso con el símbolo emblema de la paz de los años sesenta y setenta no deja ninguna duda, pareciera tratarse de un actor defensor de la causa ambiental, y cuyo único interés es el medio ambiente, lo cual se refuerza con que en esta parte de la cartilla el protagonismo ambiental lo tiene el gas natural sobre el biodiesel.

\section{Conclusiones}

Después del análisis desarrollado, con base en el seguimiento de los actores en el proceso de diseño de la cartilla, es importante destacar tres campos para futuros ejercicios de investigación que busquen identificar posibles deformaciones de la ciencia, no sólo en escenarios formales, sino también en espacios educativos noformales:

En primer lugar, es necesario pensar cuál es la idea de ciencia y tecnología que se tiene y cómo se entienden estas deformaciones a la luz del contexto específico 
de análisis; por ejemplo, para el caso colombiano, una visión elitista de la ciencia sobrepasa lo planteado por Fernández et al. (2002), y requiere incorporar aspectos relacionados con la colonialidad y la dependencia que de algún modo también configuran nuestras visiones sobre la ciencia. Este tipo de visiones elitistas llevan a pensar que Colombia, al ser un país periférico, se constituye como escenario que, antes que producir tecnología, es asumido sólo como usuario de desarrollos diseñados y producidos en otros lugares.

Esto sería promovido por la cartilla, por cuanto la contextualización del uso del gas en la ciudad está directamente asociada a desarrollos tecnológicos que son importados, sin hacer ningún énfasis en la apropiación local que se hace de éstos como modos de identificar referentes de desarrollos propios (considerando a la apropiación como una forma de re-desarrollar). Por otra parte, este énfasis en el uso y consumo de tecnología va a estar respaldado por el papel de la cartilla, como herramienta de la empresa distribuidora de gas natural, que ya hemos venido señalando.

Este llamado a contextualizar debe también ser incorporado a la noción de contexto social propuesta por Fernández et al. El análisis del caso GNE muestra la necesidad de incorporar a esta categoría, los intereses de quienes financian los materiales, que para el caso de la cartilla que ha sido de-construida, constituyen una búsqueda por posicionar una empresa en un escenario para ella emergente (los combustibles para automóviles).

En este sentido, la cartilla constituye un vehículo que dinamizará su imagen corporativa, por cuanto permite señalar los problemas y los vacíos de otros recursos energéticos, asumiendo con ello, tácitamente, que el producto que entra, en este caso el gas natural, será mejor que los otros. La cartilla GNE estaría, por medio del centro interactivo, usando la misma estrategia de marketing a la que nos tiene acostumbrados el mercado: posicionar un producto gracias a la oposición frente a otro.

En segundo lugar, la metodología propuesta por Fernández et al. $(2002,2003)$ se queda corta al analizar el producto terminado, pues desconoce las circunstancias que materializaron o modelaron algunas deformaciones, suponiendo, de alguna manera, que ellas se gestan como ignorancia o ingenuidad, desconociendo, por ejemplo, que en las circunstancias dadas en un proyecto como GNE están en juego intereses que modelan una ciencia y una tecnología que se ajusta a ellos, en este caso intereses simbólicos de generar necesidades de consumo, como lo devela el ejercicio presentado anteriormente.

Volviendo al análisis realizado a la luz de las categorías propuestas por Fernández et al., es posible afirmar que este instrumento tiende a impulsar una noción de ciencia "buena" y "justa", que no deja de levantar sospecha. No cabe ni es pertinente aquí la discusión sobre la complejidad de lo que la empresa científico-tecnológica es, más allá de lo que las deformaciones "descubren", pero sí consideramos necesario señalar que esta búsqueda por desvelar deformaciones, se convierte peligrosamente en un mecanismo para legitimar esa empresa. Asunto que esperamos haber profundizado empíricamente en este artículo. 
Por otro lado, es necesario llamar la atención sobre la importancia que tienen los centros interactivos en el posicionamiento de la ciencia y la tecnología, pero también lo es preguntarse por las visiones que construye o ayuda a reforzar sobre ella. En este sentido, el análisis muestra una relación directa de la ciencia y la tecnología con unos intereses que vienen especialmente de la empresa privada y encuentran un nicho en unas condiciones especiales facilitadas por la SED, Maloka y los maestros. Esta circunstancia no es extraña y ya nos la habían advertido Latour (1993; 1998a; 2001), Callon (1998) y Winner (1987), entre otros; sin embargo, ejercicios como el planteado permiten reconocer la presencia de estos intereses (publicitarios, instrumentales, de posicionamiento de un sector por medio de otro) en el escenario educativo y problematizarlos.

Es imperativo generar reflexiones como estas, que permitan problematizar los programas desarrollados por los centros interactivos, que, como sucede en el caso de Maloka, son ventanas para las empresas privadas, aspectos que son reconocidos por otros actores, pero que no siempre son abordados desde la academia, ejemplo de ello es lo que señala una de las maestras que participó del proyecto "[...] yo creo que Gas Natural se metió en ese cuento de Maloka porque es un ventana que visita muchos colegios, la mayoría de la gente, ahí de cierta manera Maloka es la ventana para que sea conocido el gas natural” (Docente CRH, marzo de 2008).

Por último, uno de los errores de la reflexión educativa, es tener en cuenta sólo los actores humanos y no los otros actores inmersos en el escenario educativo, que cada vez cobran mayor importancia. Parece que como pedagogos nos quedamos anclados en un escenario en que el único dueño y señor del aula somos nosotros, sin considerar que desde los cambios sustanciales ocurridos en la educación en la década del noventa, incluso antes, y las posibilidades de acceso a la información por las llamadas nuevas tecnologías, el papel de la enseñanza ha sido subordinado a la responsabilidad educativa sobre el "aprendizaje” (Martínez, 2004, p. 401).

Esta nueva configuración del escenario educativo abre la puerta a un sinnúmero de herramientas que articulan una serie de intereses más allá de los propiamente educativos (económicos, políticos y sociales). Éstos, llamados por la TAR como nohumanos, esconden relaciones de poder, ya que cuando se actúa con ellos (computadores, material didáctico, textos escolares, centros interactivos, etc.) la dominación y la exclusión se ocultan bajo la idea de fuerzas objetivas y naturales (Latour, 1998, p. 283) ${ }^{12}$.

\footnotetext{
${ }^{12}$ Mirar las cifras y el interés que se presta a los no-humanos nos da una idea de la dimensión de su protagonismo. En Colombia, por ejemplo, se imprimieron en 2006, del orden de $10^{\prime} 000.000$ de libros escolares (Cámara Colombiana del Libro, 2007), 54.000 computadores anuales se entregarán por el programa Computadores para Educar entre 2007 y 2010 (Ministerio de Educación Nacional, 2007), los programas de formación virtual del Sena comenzaron en 2003, con 27.700 estudiantes, pasaron a 129.630 en 2004 y a 231.000 en lo corrido de enero a octubre de 2005 (Presidencia de la República, 2006). COLCIENCIAS ha invertido en el periodo 1995-2005 en Maloka una suma que supera los ocho mil millones de pesos (Daza et ál., 2006) y la Alcaldía de Medellín invirtió 72.000 millones de pesos en el centro interactivo Explora, centro interactivo ubicado en esta ciudad (Alcaldía de Medellín-Compromiso, 2007).
} 


\section{Referencias bibliográficas}

Abd-El-Khalick, F. y Lederman, N. (2000). Improving science teachers' conceptions of nature of science: a critical review of the literature. International Journal of Science Education, 7(22), 665701.

Acevedo Díaz, J. y Acevedo RomeRO, P. (2002). Creencias sobre la naturaleza de la ciencia. Un estudio con titulados universitarios en formación inicial para ser profesores de educación secundaria. Revista Iberoamericana de Educación, obtenido el 5 de mayo de 2007, desde http://www.rieoei.org/ deloslectores/244Acevedo.PDF

\section{Alcaldía de Medellín-Compromiso} (2007). El parque explora, ya recibe a sus primeros exploradores. Obtenido el 10 de febrero de 2008, desde http://www. medellin.gov.co

Aguirre, J. (ED.). (2005). La percepción que tienen los colombianos sobre la ciencia y la tecnología. Bogotá: Colciencias.

Ayús, R. (2002). Estudios sociales de ciencia y tecnología: merodeando en el campo. Organización de Estados Iberoamericanos para la Educación, la Ciencia y la Cultura (CTS+I). Obtenido el 10 de febrero de 2008, desde http://www. campus-oei.org/salactsi/ramfis.htm

Callon, M. (1998). El proceso de construcción de la sociedad. El estudio de la tecnología como herramienta para el análisis sociológico. En M. Domènech y F. Tirado (comp.) (s. f.), Sociología simétrica, ensayos sobre ciencia, tecnología y sociedad. Barcelona: Gedisa.
Cámara Colombiana del Libro. (2007). Estadísticas del libro en Colombia 2002-2006. Obtenido el 10 de febrero de 2008, desde http://www.camlibro.com.co

Daza, S. y Arboleda, T. (2007). Comunicación pública de la ciencia y la tecnología en Colombia: ¿políticas para la democratización del conocimiento?. Revista Signo y Pensamiento, 50.

DAzA, S. et al. (2006). Evaluación de las actividades de comunicación pública de la ciencia y la tecnología en el sistema nacional de ciencia y tecnología colombiano 1990-2004. Bogotá: Observatorio Colombiano de Ciencia y Tecnología para Colciencias.

Fernández, I., Gil, D., Carrascosa, J., Cachapuz, A. y Praia, J. (2002). Visiones deformadas de la ciencia transmitidas por la enseñanza. Enseñanza de las Ciencias, 3(20), 477-488.

Fernández, I., Gil, D., Vilches, A., Valdés, P., Cachapuz, A., Praia, J. y SaliNAS, J. (2003). El olvido de la tecnología como refuerzo de las visiones deformadas de la ciencia. Revista Electrónica de Enseñanza de las Ciencias vol., 3.

Gallego Torres, P. (2007). Imagen popular de la ciencia transmitida por los cómics. Revista Eureka: sobre Enseñanza y Divulgación de las Ciencias, 1(4), 141151. Obtenido el 10 de mayo de 2007 , desde http://www.apac-eureka.org/revista

Harding, S. (1991). Whose science? Whose knowledge? Nueva York: Cornell University Press. 
Latour, B. (1993), We have never been modern. Cambridge-Massachusetts: Harvard University Press.

LATOur, B. (1998a). De la mediación técnica: filosofía, sociología, genealogía. En M. Domènech y F. Tirado (comp.) (s. f.), Sociología simétrica, ensayos sobre ciencia, tecnología y sociedad. Barcelona: Gedisa.

LATOur, B. (1998b). Visualización y cognición: pensando con los ojos y con las manos. La balsa de la medusa, 45-46, 77-126.

Latour, B. (2001). La esperanza de Pandora, ensayos sobre la realidad de los estudios de la ciencia. Barcelona: Gedisa.

Martínez, A. (2004), De la escuela expansiva a la escuela competitiva: dos modos de modernización en América Latina. Barcelona: Anthropos.

Ministerio de Educación Nacional. (2007). 2007 año del salto en inversión para tecnologías de la información en educación. Obtenido el 10 de febrero de
2008, desde: http://www.mineducacion. gov.co/cvn/1665/printer-115871.html

Osorio, C. (2005). La participación pública en sistemas tecnológicos. Lecciones para la educación CTS. Revista CTS, 6(2), 159-172. Obtenido el 19 de abril de 2008, desde http://www.revistacts.net/2/6/ dossier6/file

Pinch, T. (1997). La construcción social de la tecnología: una revisión. En J. Santos y D. Rodrigo (comp.) (s. f.), Innovación tecnológica y procesos culturales. Nuevas perspectivas teóricas. México: Ediciones Científicas UniversitariasUnam-Fondo de Cultura Económica.

Presidencia de la República. (2006). Sena expondrá resultados de educación virtual en Estados Unidos. Obtenido el 10 de febrero de 2008, desde http:// www.presidencia.gov.co/sne/2006/ enero/02/13022006.htm

WiNNER, L. (1987). La ballena y el reactor. Barcelona: Gedisa. 
